\title{
Two New Acylated Flavanone Glycosides from the Leaves and Branches of Phyllanthus emblica
}

\author{
Ying-Jun Zhang, ${ }^{a}$ Tomomi Abe, ${ }^{a}$ Takashi Tanaka, ${ }^{a}$ Chong-Ren YAng, ${ }^{b}$ and Isao Kouno*, $a$ \\ ${ }^{a}$ Faculty of Pharmaceutical Sciences, Nagasaki University; 1-14 Bunkyo-Machi, Nagasaki 852-8521, Japan: and \\ ${ }^{b}$ Laboratory of Phytochemistry, Kunming Institute of Botany, Chinese Academy of Sciences; Kunming 650204, P. R. China. \\ Received November 28, 2001; accepted February 18, 2002
}

Two new acylated flavanone glycosides, $(S)$-eriodictyol 7-O-(6"-O-trans-p-coumaroyl)- $\beta$-D-glucopyranoside (1) and $(S)$-eriodictyol 7-O-(6"-O-galloyl)- $\beta$-D-glucopyranoside (2) were isolated from the leaves and branches of Phyllanthus emblica together with a new phenolic glycoside, 2-(2-methylbutyryl)phloroglucinol 1-O-(6"-O- $\beta$-Dapiofuranosyl)- $\beta$-D-glucopyranoside (3), as well as 22 known compounds. Their structures were determined by spectral and chemical methods.

Key words Phyllanthus emblica; Euphorbiaceae; acylated flavanone glycoside

Phyllanthus emblica L. (Euphorbiaceae) is a shrub or tree native to subtropical and tropical areas of China, India, Indonesia and the Malay Peninsula. The fruit has been widely used for antiinflammatory and antipyretic treatment. The root, leaves and bark are also used for the treatment of indigestion, diarrhea or dysentery, eczema and wart. As a continuation of investigation on the constituents of this plant, ${ }^{1-5}$ ) we chemically examined its leaves and branches, and two new acylated flavanone glycosides, a new phenolic glycoside, and 22 known compounds were isolated. This paper describes the isolation and structural elucidation of these compounds.

\section{Results and Discussion}

As described in the previous paper, ${ }^{2)}$ the EtOH extract of the fresh leaves and branches of $P$. emblica was suspended in water and then extracted with $\mathrm{Et}_{2} \mathrm{O}$. The $\mathrm{Et}_{2} \mathrm{O}$ layer was partitioned between hexane and $\mathrm{MeOH}$, and the $\mathrm{MeOH}$ layer was further chromatographed successively over Sephadex LH-20, silica gel, MCI-gel CHP 20P and Chromatorex ODS to afford $\mathbf{1}$ and $\mathbf{2}$, as well as 17 known compounds. The known ones were identified as naringenin, ${ }^{6)}$ eriodictyol, ${ }^{6}$ kaempferol, ${ }^{7)}$ dihydrokaempferol, ${ }^{8,9)}$ quercetin, ${ }^{7)}$ naringenin 7-O-glucoside (prunin), ${ }^{10)}$ naringenin 7-O-(6"-O-galloyl)-glucoside, ${ }^{10)}$ naringenin 7-O-(6"-O-trans- $p$-coumaroyl)-glucoside, ${ }^{11)}$ kaempferol 3-O-rhamnoside, ${ }^{7)}$ quercetin 3-O-rhamnoside, ${ }^{7)}$ myricetin 3-O-rhamnoside, ${ }^{7)}$ 2-(2-methylbutyryl)phloroglucinol 1- $O$ - $\beta$-D-glucopyranoside (multifidol glucoside) $(5),{ }^{12)}(-)$-epigallocatechin 3- $O$-gallate, ${ }^{13)}$ 1,2,3,6-tetra$O-{ }^{14)} 1,2,4,6$-tetra- $O$-, ${ }^{15)}$ and $1,2,3,4,5$-penta- $O$-galloyl- $\beta$-Dglucose, ${ }^{16)}$ and decarboxyellagic acid ${ }^{17)}$ by comparison of the physical and spectral data with literature values.

The water layer was separated first by Sephadex LH-20 column chromatography, ${ }^{2}$ and the obtained fraction 1 was subjected to MCI-gel CHP 20P, Chromatorex ODS, and silica gel to afford $\mathbf{3}$, together with 8 known constituents identified as eriodictyol 7-O-glucoside (4), ${ }^{18)}$ kaempferol 3-Orhamnoside, quercetin 3-O-rhamnoside, quercetin 3-O-glucoside ${ }^{7}{ }^{7}$ myricetin 3-O-rhamnoside, rutin, ${ }^{19)} 3-O$-methylellagic acid $4^{\prime}-O-\alpha$-L-rhamnopyranoside ${ }^{20)}$ and tuberonic (12-OHjasmonic) acid glucoside. ${ }^{21)}$ The last compound was first isolated from the potato leaves as a tuber-inducing stimulus ${ }^{22}$ ) and found to be a leaf-closing substance of Albizzia julib- rissin. ${ }^{21)}$ This is the first time that tuberonic acid glucoside was obtained from $P$. emblica, which has a nyctinastic movement.

Compound 1, a yellow amorphous powder, had a molecular formula $\mathrm{C}_{30} \mathrm{H}_{28} \mathrm{O}_{13}$ on the basis of its ${ }^{13} \mathrm{C}$-NMR spectral data (Table 1), negative-ion FAB-MS $\left[\mathrm{m} / \mathrm{z} 595,(\mathrm{M}-\mathrm{H})^{-}\right]$ and elemental analysis. The ${ }^{1} \mathrm{H}$ - and ${ }^{13} \mathrm{C}$-NMR spectra of $\mathbf{1}$ were closely related to those of eriodictyol 7-O-glucoside $(4),{ }^{18)}$ except for the appearance of additional signals $[\delta 7.52$, 6.35 (each d, $J=16.0 \mathrm{~Hz}$ ) and $\delta 7.49,6.79$ (each $2 \mathrm{H}$, d, $J=8.5 \mathrm{~Hz}$ )] arising from a trans- $p$-coumaroyl group. Acidic hydrolysis of $\mathbf{1}$ in aqueous $\mathrm{MeOH}$ yielded eriodictyol, D-glucose and coumaric acid methyl ester, confirming the components of the molecule 1 . The location of the $p$-coumaroyl was determined to be the glucose C- 6 " position on the basis of the downfield shift of the glucose C-6" ( $\delta$ 63.3) and H-6" $\delta 4.42$ (dd, $J=2.0,12.0 \mathrm{~Hz}$ ) and $4.14(\mathrm{dd}, J=6.5,12.0 \mathrm{~Hz})]$ by comparison with those of eriodictyol $7-O$-glucoside $\left[\delta_{\mathrm{C}} 60.8 ; \delta_{\mathrm{H}}\right.$ $4.03,3.85]$. The linkage of the glucopyranosyl moiety at the 7-hydroxyl group of eriodictyol was confirmed by the HMBC correlation between the glucose $\mathrm{H}-1^{\prime \prime}(\delta 5.05$, d, $J=7.5 \mathrm{~Hz})$ and $\mathrm{C}-7$ ( $\delta$ 165.1). The absolute stereochemistry at C-2 was assigned to be $S$ by observation of a positive Cotton effect at $337 \mathrm{~nm}$ and a negative one at $294 \mathrm{~nm}$ in the cir-

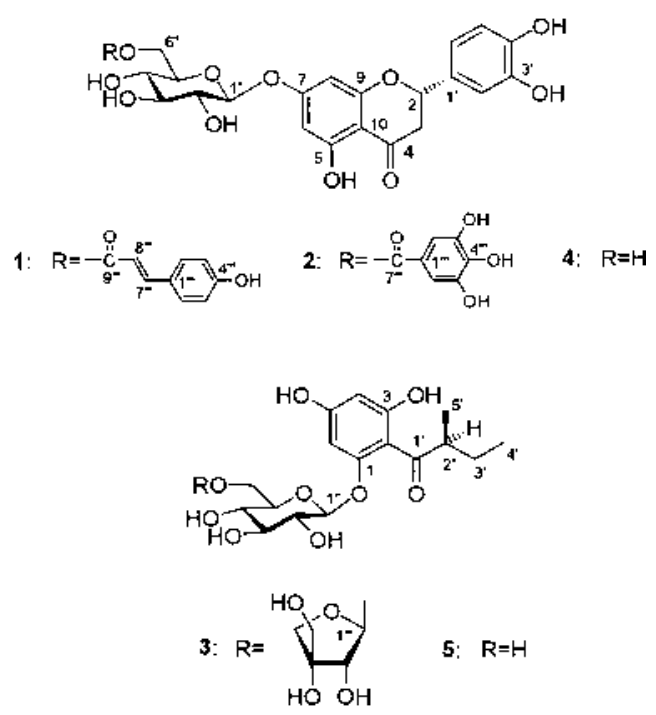

(C) 2002 Pharmaceutical Society of Japan 
Table $1 .{ }^{13} \mathrm{C}$-NMR Data of Compounds $\mathbf{1}-\mathbf{5}$

\begin{tabular}{|c|c|c|c|c|c|c|}
\hline & $\mathbf{1}^{a)}$ & $2^{a)}$ & $4^{b)}$ & & $3^{c)}$ & $\mathbf{5}^{d)}$ \\
\hline C-2 & 78.6 & 78.7 & 78.9 & C-1 & 161.8 & 161.7 \\
\hline 3 & 42.2 & 42.2 & 44.4 & 2 & 106.9 & 106.5 \\
\hline 4 & 197.1 & 197.1 & 197.4 & 3 & 165.5 & 166.6 \\
\hline 5 & 163.1 & 163.1 & 163.2 & 4 & 95.6 & 95.7 \\
\hline 6 & 95.7 & 95.3 & 95.7 & 5 & 167.3 & 166.4 \\
\hline 7 & 165.1 & 165.1 & 165.5 & 6 & 98.4 & 98.6 \\
\hline 8 & 96.3 & 96.3 & 96.7 & $1^{\prime}$ & 211.8 & 211.7 \\
\hline 9 & 162.6 & 162.8 & 162.9 & $2^{\prime}$ & 46.9 & 46.8 \\
\hline 10 & 103.4 & 103.4 & 103.5 & $3^{\prime}$ & 28.3 & 28.2 \\
\hline $1^{\prime}$ & 129.3 & 129.3 & 129.4 & $4^{\prime}$ & 12.1 & 12.0 \\
\hline $2^{\prime}$ & 114.3 & 114.4 & 114.7 & $5^{\prime}$ & 16.8 & 16.8 \\
\hline $3^{\prime}$ & 145.0 & 145.3 & 145.4 & glu-1" & 101.7 & 101.5 \\
\hline $4^{\prime}$ & 145.8 & 145.9 & 146.0 & $2^{\prime \prime}$ & 74.8 & 74.7 \\
\hline $5^{\prime}$ & 115.4 & 115.4 & 115.6 & $3^{\prime \prime}$ & $78.6^{f)}$ & 78.6 \\
\hline $6^{\prime}$ & 118.1 & 118.3 & 118.4 & $4^{\prime \prime}$ & 71.3 & 71.1 \\
\hline glu-1" & 99.4 & 99.4 & 99.8 & $5^{\prime \prime}$ & $78.0^{f)}$ & 78.2 \\
\hline $2^{\prime \prime}$ & 73.0 & 73.1 & 73.2 & $6^{\prime \prime}$ & 68.6 & 62.4 \\
\hline $3^{\prime \prime}$ & 76.2 & 76.1 & $77.3^{e)}$ & api-1"' & 110.9 & \\
\hline $4^{\prime \prime}$ & 69.9 & 69.3 & 69.7 & $2^{\prime \prime \prime}$ & 77.2 & \\
\hline $5^{\prime \prime}$ & 73.9 & 73.8 & $76.5^{e)}$ & $3^{\prime \prime \prime}$ & 80.5 & \\
\hline $6^{\prime \prime}$ & 63.3 & 63.0 & 60.8 & $4^{\prime \prime \prime}$ & 75.0 & \\
\hline Acyl group & & & & $5^{\prime \prime \prime}$ & 65.7 & \\
\hline $1^{\prime \prime \prime}$ & 125.1 & 119.4 & & & & \\
\hline $2^{\prime \prime \prime}, 6^{\prime \prime \prime}$ & 130.3 & 108.8 & & & & \\
\hline $3^{\prime \prime \prime}, 5^{\prime \prime \prime}$ & 115.9 & 145.6 & & & & \\
\hline $4^{\prime \prime \prime}$ & 159.9 & 138.6 & & & & \\
\hline $7^{\prime \prime \prime}$ & 145.3 & 165.9 & & & & \\
\hline $8^{\prime \prime \prime}$ & 114.0 & & & & & \\
\hline $9^{\prime \prime \prime}$ & 166.5 & & & & & \\
\hline
\end{tabular}

cular dichroism (CD) spectrum. ${ }^{23,24)}$ Therefore, the structure of 1 was established as $(S)$-eriodictyol 7-O-(6"-O-trans- $p$ coumaroyl)- $\beta$-D-glucopyranoside.

Compound $\mathbf{2}$ was obtained as a yellow amorphous powder and gave dark blue coloration with the ferric chloride reagent. Comparison of the ${ }^{1} \mathrm{H}$ - and ${ }^{13} \mathrm{C}$-NMR spectral data (Table 1) with those of $\mathbf{1}$ showed that their structures were very similar except for the presence of a galloyl group in $\mathbf{2}$ instead of the coumaroyl group in $\mathbf{1}$. The presence of the galloyl group in $\mathbf{2}$ could be easily recognized from the characteristic two-proton singlet at $\delta 6.91$ and seven $s p^{2}$ carbon signals $[\delta 145.6(2 \mathrm{C}), 138.6,119.4,108.8(2 \mathrm{C}), 165.9]$ in the ${ }^{1} \mathrm{H}$ - and ${ }^{13} \mathrm{C}$-NMR spectra. Acidic hydrolysis of $\mathbf{2}$ yielded eriodictyol, glucose and gallic acid, confirming that $\mathbf{2}$ was a galloyl ester of eriodictyol glucoside. The location of the ester was determined to be at glucose C- 6 " on the basis of the long-range correlations between the two methylene protons of glucose H-6" and the galloyl carboxyl carbon ( $\delta$ 165.9) signals in the heteronuclear multiple bond connectivity (HMBC) spectrum. In addition, the HMBC correlation of the glucose anomeric proton $(\delta 5.08, \mathrm{~d}, J=7.5 \mathrm{~Hz})$ with $\mathrm{C}-7(\delta$ $165.1)$ confirmed that the $6 "-O$-galloylglucopyranosyl moiety was attached to the 7-hydroxyl group of eriodictyol. The absolute stereochemistry at C-2 was also assigned as $S$ based on a positive Cotton effect at $336 \mathrm{~nm}$ and a negative one at $291 \mathrm{~nm}$ in the CD spectrum. Thus, the structure of 2 was determined as $(S)$-eriodictyol 7-O-(6"-O-galloyl)- $\beta$-D-glucopyranoside.

Compound 3 had a molecular formula $\mathrm{C}_{22} \mathrm{H}_{32} \mathrm{O}_{13}$ on the basis of the ${ }^{13} \mathrm{C}-\mathrm{NMR}$ spectral data (Table 1), the negativeion FAB-MS $\left[m / z 503,(\mathrm{M}-\mathrm{H})^{-}\right]$and elemental analysis.
The ${ }^{1} \mathrm{H}$ - and ${ }^{13} \mathrm{C}-\mathrm{NMR}$ spectral data of $\mathbf{3}$ were similar to those of 2-(2-methylbutyryl)phloroglucinol (multifidol) glucoside $(5)^{12)}$ except for the appearance of a set of signals arising from a pentose moiety. The ${ }^{1} \mathrm{H}$ - and ${ }^{13} \mathrm{C}$-NMR spectral data (Table 1) due to the sugar moieties revealed the existence of a $6-O$ - $\beta$-apiofuranosyl- $\beta$-glucopyranosyl moiety in $3^{25)}$ Furthermore, hydrolysis of $\mathbf{3}$ yielded 2-(2-methylbutyryl)phloroglucinol (multifidol), ${ }^{12)} \mathrm{D}$-glucose $\left([\alpha]_{\mathrm{D}}+24.3^{\circ}\right)$ and D-apiose $\left([\alpha]_{\mathrm{D}}+6.5^{\circ}\right)$. On the basis of the above results, 3 was characterized as 2-(2-methylbutyryl)phloroglucinol $1-O$-(6"- $O$ - $\beta$-D-apiofuranosyl)- $\beta$-D-glucopyranoside. The absolute configuration of C-2' was assigned as $S$ according to the positive Cotton effect at $280 \mathrm{~nm}$ in the CD spectrum. ${ }^{12}$ )

Up to now, we have studied the chemical constituents of the roots, fruit juice, and leaves and branches of $P$. emblica, ${ }^{1-5)}$ and obtained a number of polyphenols and sesquiterpenoids which might be the potent bioactive principles of the plant. Studies of the antioxidative and antiproliferative activities of these components are now in progress.

\section{Experimental}

The instruments used for obtaining physical data and experimental conditions for chromatography were the same as we described previously. ${ }^{2-5)}$

Plant Material The leaves and branches of Phyllanthus emblica were collected at Xishuangbanna, Yunnan, the People's Republic of China. A voucher specimen is deposited in the Herbarium of Kunming Institute of Botany, Chinese Academy of Sciences.

Extraction and Isolation As described in the previous paper, ${ }^{2)}$ the EtOH extract of the fresh leaves and branches $(15 \mathrm{~kg})$ of $P$. emblica was suspended in water and then partitioned with $\mathrm{Et}_{2} \mathrm{O}$. After being concentrated to dryness, the $\mathrm{Et}_{2} \mathrm{O}$ layer $(198.0 \mathrm{~g})$ was further partitioned between hexane and $\mathrm{MeOH}$. The MeOH layer (100.34 g) was chromatographed over Sephadex LH-20 $(80-100 \% \mathrm{MeOH}$, and then $50 \%$ acetone) to give four fractions 
(fractions $1-4)$. Fraction $2(68.3 \mathrm{~g})$ was subjected to a silica gel $\left(\mathrm{CH}_{3} \mathrm{Cl}-\right.$ $\mathrm{MeOH}-\mathrm{H}_{2} \mathrm{O}, 100: 0: 0-5: 5: 1$ ) column and afforded seven fractions (fractions 2-1-2-7), among which fraction 2-5 (5.05 g) was identified as quercetin 3-O-rhamnoside. Fractions 2-1-2-4 and 2-6-2-7 were separately chromatographed over Sephadex LH-20 (40-90\% MeOH) and MCIgel CHP 20P $(0-100 \% \mathrm{MeOH})$ to give kaempferol $(6.5 \mathrm{mg})$, dihydrokaempferol $(12.9 \mathrm{mg})$, naringenin $(25.2 \mathrm{mg})$ and eriodictyol $(17.5 \mathrm{mg})$ from fraction $2-1$, quercetin $(63 \mathrm{mg})$ from fraction $2-2$, naringenin $7-O-\left(6^{\prime \prime}-\right.$ $O$-trans- $p$-coumaroyl)-glucoside $(167 \mathrm{mg})$ from fraction $2-3,1$ (160 mg), decarboxyellagic acid $(105 \mathrm{mg}), \mathbf{5}(221 \mathrm{mg})$, naringenin $7-O$-glucoside (77 $\mathrm{mg})$, gallic acid $(1.37 \mathrm{~g})$ and kaempferol 3-O-rhamnoside $(846 \mathrm{mg})$ from fraction 2-4, 2 (95 mg), naringenin 7-O-(6"-O-galloyl)-glucoside $(35 \mathrm{mg})$, myricetin 3-O-rhamnoside $(20 \mathrm{mg}),(+)$-gallocatechin $(7.9 \mathrm{mg}),(-)$-epicagallocatechin $(6.6 \mathrm{mg})$ and $(-)$-epigallocatechin $3-O$-gallate $(17.6 \mathrm{mg})$ from fraction $2-6$, and 1,2,3,6-tetra- $O$-galloyl- $\beta$-D-glucose $(176 \mathrm{mg}), 1,2,4,6$-tetra$O$-galloyl- $\beta$-D-glucose $(75 \mathrm{mg}$ ), and $1,2,3,4,6$-penta- $O$-galloyl- $\beta$-D-glucose (45 mg) from fraction $2-7$, respectively.

The water layer was treated as described ${ }^{2)}$ to give five fractions (fractions $1-5)$. Fraction 1 was subjected to MCI-gel CHP 20P $(0-100 \% \mathrm{MeOH})$, Chromatorex ODS $(40-100 \% \mathrm{MeOH})$ and $\mathrm{Si}$ gel $\left(\mathrm{CHCl}_{3}-\mathrm{MeOH}-\mathrm{H}_{2} \mathrm{O}\right.$, $9: 1: 0.1-6: 4: 1)$ to afford $3(32.7 \mathrm{mg}), 3^{\prime}-O$-methylellagic acid $4-\mathrm{O}-\alpha$-Lrhamnopyranoside $(91 \mathrm{mg}), 4$ (156 mg), kaempferol 3- $O$-rhamnoside (200 $\mathrm{mg})$, quercetin 3 - $O$-rhamnoside $(402 \mathrm{mg})$, quercetin 3 - $O$-glucoside (176 $\mathrm{mg})$, myricetin $3-O$-rhamnoside $(17 \mathrm{mg})$, rutin $(12 \mathrm{mg})$ and tuberonic acid glucoside $(556 \mathrm{mg})$.

$(S)$-Eriodictyol 7-O-(6"-O-trans- $p$-Coumaroyl)- $\beta$-D-glucopyranoside (1): Yellow amorphous powder, $[\alpha]_{\mathrm{D}}^{28}-92.8^{\circ} \quad(c=0.36, \mathrm{MeOH})$. UV $\lambda_{\max }$ $(\mathrm{EtOH}) \mathrm{nm}(\log \varepsilon): 286(4.27), 315$ (4.48). CD $\lambda_{\max }(\mathrm{EtOH}) \mathrm{nm}(\Delta \varepsilon): 337$ $(+4.1), 294(-29.2) .{ }^{1} \mathrm{H}-\mathrm{NMR}\left(\mathrm{DMSO}-d_{6}\right): \delta: 2.71 \quad(1 \mathrm{H}, \mathrm{dd}, J=3.0$, $17.0 \mathrm{~Hz}, \mathrm{H}-3 \mathrm{~b}), 3.21$ (1H, dd, $J=12.0,17.0 \mathrm{~Hz}, \mathrm{H}-3 \mathrm{a}), 3.27(1 \mathrm{H}, \mathrm{dd}, J=7.5$, $\left.8.5 \mathrm{~Hz}, \mathrm{H}-2^{\prime \prime}\right), 3.33$ (1H, dd, $\left.J=8.5,9.0 \mathrm{~Hz}, \mathrm{H}-3^{\prime \prime}\right), 3.36-3.58$ (1H, overlapping with solvent, H-4"), $3.75\left(1 \mathrm{H}\right.$, ddd, $\left.J=2.0,6.5,9.0 \mathrm{~Hz}, \mathrm{H}-5^{\prime \prime}\right), 4.14(1 \mathrm{H}$, dd, $\left.J=6.5,12.0 \mathrm{~Hz}, \mathrm{H}-6^{\prime \prime} \mathrm{b}\right), 4.42$ (1H, dd, $\left.J=2.0,12.0 \mathrm{~Hz}, \mathrm{H}-6^{\prime \prime} \mathrm{a}\right), 5.05$ (1H, d, $\left.J=7.5 \mathrm{~Hz}, \mathrm{H}-1^{\prime \prime}\right), 5.37(1 \mathrm{H}, \mathrm{dd}, J=3.0,12.0 \mathrm{~Hz}, \mathrm{H}-2), 6.12(1 \mathrm{H}, \mathrm{d}$, $J=2.5 \mathrm{~Hz}, \mathrm{H}-6), 6.18(1 \mathrm{H}, \mathrm{d}, J=2.5 \mathrm{~Hz}, \mathrm{H}-8), 6.35(1 \mathrm{H}, \mathrm{d}, J=16.0 \mathrm{~Hz}$, H-8'"'), 6.73 (2H, br s, H-5', 6'), $6.79\left(2 \mathrm{H}, \mathrm{d}, J=8.5 \mathrm{~Hz}, \mathrm{H}-3^{\prime \prime \prime}, 5^{\prime \prime \prime}\right), 6.87(1 \mathrm{H}$, d, $\left.J=2.0 \mathrm{~Hz}, \mathrm{H}-2^{\prime}\right), 7.49\left(2 \mathrm{H}, \mathrm{d}, J=8.5 \mathrm{~Hz}, \mathrm{H}-2^{\prime \prime \prime}, 6^{\prime \prime \prime}\right), 7.52(1 \mathrm{H}, \mathrm{d}$, $\left.J=16.0 \mathrm{~Hz}, \mathrm{H}-7^{\prime \prime \prime}\right), 12.05(1 \mathrm{H}, \mathrm{s}, 5-\mathrm{OH}) .{ }^{13} \mathrm{C}-\mathrm{NMR}$ : see Table 1. FAB-MS $m / z: 595(\mathrm{M}-\mathrm{H})^{-}$. Anal. calcd for $\mathrm{C}_{30} \mathrm{H}_{28} \mathrm{O}_{13} \cdot 2 \mathrm{H}_{2} \mathrm{O}: \mathrm{C}, 56.96 ; \mathrm{H}, 5.10$. Found: C, 57.12; H, 5.12.

Hydrolysis of 1 A solution of $\mathbf{1}(20 \mathrm{mg})$ in $\mathrm{MeOH}(1 \mathrm{ml})$ was treated with $1 \mathrm{M} \mathrm{H}_{2} \mathrm{SO}_{4}(3 \mathrm{ml})$ at $80^{\circ} \mathrm{C}$ for $3 \mathrm{~h}$. The reaction mixture was extracted with $\mathrm{Et}_{2} \mathrm{O}$ and the $\mathrm{Et}_{2} \mathrm{O}$ layer was applied to Sephadex LH-20 and silica gel to afford $p$-coumaric acid methyl ester $(1 \mathrm{mg}),{ }^{1} \mathrm{H}-\mathrm{NMR}\left(\mathrm{CD}_{3} \mathrm{OD}\right): \delta: 3.62$ (3H, s, OMe), 6.23 (1H, d, $J=16.2 \mathrm{~Hz}, \mathrm{H}-8), 6.71$ (2H, d, $J=8.7 \mathrm{~Hz}, \mathrm{H}-3,5)$, $7.36(2 \mathrm{H}, \mathrm{d}, J=8.7 \mathrm{~Hz}, \mathrm{H}-2,6), 7.52(1 \mathrm{H}, \mathrm{d}, J=16.2 \mathrm{~Hz}, \mathrm{H}-7)$; and eriodictyol $^{11)}(6.5 \mathrm{mg}),{ }^{1} \mathrm{H}-\mathrm{NMR}\left(\mathrm{CD}_{3} \mathrm{OD}\right): \delta: 2.69(1 \mathrm{H}, \mathrm{dd}, J=3.0,17.1 \mathrm{~Hz}, \mathrm{H}-3 \mathrm{a})$, $3.07(1 \mathrm{H}, \mathrm{dd}, J=12.6,17.1 \mathrm{~Hz}, \mathrm{H}-3 \mathrm{~b}), 5.28$ (1H, dd, $J=3.0,12.6 \mathrm{~Hz}, \mathrm{H}-2)$, $5.87(1 \mathrm{H}, \mathrm{d}, J=2.1 \mathrm{~Hz}, \mathrm{H}-6), 5.89(1 \mathrm{H}, \mathrm{d}, J=2.1 \mathrm{~Hz}, \mathrm{H}-8), 6.78$ (2H, br s, H$\left.5^{\prime}, 6^{\prime}\right), 6.91(1 \mathrm{H}$, br s, H-2'). The water layer was neutralized with Amberlite IRA-400 ( $\mathrm{OH}^{-}$form) resin, concentrated to dryness and applied to a silica gel column to afford D-glucose $(2.4 \mathrm{mg})$ : $[\alpha]_{\mathrm{D}}^{25}+16.3^{\circ}\left(c=0.19, \mathrm{H}_{2} \mathrm{O}\right)$.

$(S)$-Eriodictyol 7-O-(6"-O-Galloyl)- $\beta$-D-glucopyranoside (2): Yellow amorphous powder, $[\alpha]_{\mathrm{D}}^{28}-79.4^{\circ}(c=0.41, \mathrm{MeOH})$. UV $\lambda_{\max }(\mathrm{EtOH}) \mathrm{nm}(\log \varepsilon)$ : 281 (4.38). CD $\lambda_{\max }(\mathrm{EtOH}) \mathrm{nm}(\Delta \varepsilon): 336(+3.1), 291(-33.3) .{ }^{1} \mathrm{H}-\mathrm{NMR}$ (DMSO-d $): \delta: 2.74(1 \mathrm{H}, \mathrm{dd}, J=3.5,17.0 \mathrm{~Hz}, \mathrm{H}-3 \mathrm{~b}), 3.24(1 \mathrm{H}, \mathrm{dd}, J=12.5$, $17.0 \mathrm{~Hz}, \mathrm{H}-3 \mathrm{a}), 3.30$ (1H, dd, $\left.J=7.5,8.5 \mathrm{~Hz}, \mathrm{H}-2^{\prime \prime}\right), 3.35(1 \mathrm{H}, \mathrm{t}, J=8.5 \mathrm{~Hz}$, H-3"), $3.38-3.48(1 \mathrm{H}$, overlapping with solvent, H-4"), $3.77(1 \mathrm{H}$, br s, H$\left.5^{\prime \prime}\right), 4.27\left(1 \mathrm{H}, \mathrm{dd}, J=4.5,12.0 \mathrm{~Hz}, \mathrm{H}-6^{\prime \prime}\right), 4.41\left(1 \mathrm{H}\right.$, br d, $\left.J=12.0 \mathrm{~Hz}, \mathrm{H}-6^{\prime \prime}\right)$, $5.08\left(1 \mathrm{H}, \mathrm{d}, J=7.5 \mathrm{~Hz}, \mathrm{H}-1^{\prime \prime}\right), 5.43(1 \mathrm{H}, \mathrm{dd}, J=3.5,12.5 \mathrm{~Hz}, \mathrm{H}-2), 6.14$ $(1 \mathrm{H}, \mathrm{d}, J=2.5 \mathrm{~Hz}, \mathrm{H}-6), 6.15(1 \mathrm{H}, \mathrm{d}, J=2.5 \mathrm{~Hz}, \mathrm{H}-8), 6.75(2 \mathrm{H}, \mathrm{brs}$, H-5', 6' $), 6.88$ (1H, br s, H-2' $), 6.91\left(2 \mathrm{H}, \mathrm{s}, \mathrm{H}-2^{\prime \prime \prime}, 6^{\prime \prime \prime}\right), 12.00(1 \mathrm{H}, \mathrm{s}, 5-\mathrm{OH})$. ${ }^{13} \mathrm{C}-\mathrm{NMR}$ : see Table 1. FAB-MS $m / z$ : $601(\mathrm{M}-\mathrm{H})^{-}$; Anal. calcd for $\mathrm{C}_{28} \mathrm{H}_{26} \mathrm{O}_{15} \cdot 5 / 2 \mathrm{H}_{2} \mathrm{O}: \mathrm{C}, 51.94 ; \mathrm{H}, 4.83$. Found: $\mathrm{C}, 51.64 ; \mathrm{H}, 4.70$.

Hydrolysis of 2 A solution of $2(1 \mathrm{mg})$ in $1 \mathrm{M} \mathrm{H}_{2} \mathrm{SO}_{4}(1 \mathrm{ml})$ was heated at $80^{\circ} \mathrm{C}$ for $3 \mathrm{~h}$, and the reaction mixture was treated in the manner described for hydrolysis of 1 . TLC analysis indicated the presence of D-glucose $\left[\mathrm{CHCl}_{3}-\mathrm{MeOH}-\mathrm{H}_{2} \mathrm{O}, 7: 3: 0.5(R f 0.1)\right.$ and benzene-ethyl formate-formic acid, $1: 7: 1(R f 0.1)]$ and gallic acid $\left[\mathrm{CHCl}_{3}-\mathrm{MeOH}-\mathrm{H}_{2} \mathrm{O}, 7: 3: 0.5(R f\right.$ $0.25)$ and benzene-ethyl formate-formic acid, $1: 7: 1(R f 0.8)]$ in the water layer, and eriodictyol $\left[\mathrm{CHCl}_{3}-\mathrm{MeOH}-\mathrm{H}_{2} \mathrm{O}, 8: 2: 0.2(R f 0.7)\right]$ in the $\mathrm{Et}_{2} \mathrm{O}$ layer by comparison with the authentic samples.

2-(2-Methylbutyryl)phloroglucinol $1-O-\left(6^{\prime \prime}-O-\beta\right.$-D-Apiofuranosyl)- $\beta$-Dglucopyranoside (3): Yellow amorphous powder, $[\alpha]_{\mathrm{D}}^{18}-60.3^{\circ}(c=0.30$,
$\mathrm{MeOH}) . \mathrm{UV} \lambda_{\max }(\mathrm{MeOH}) \mathrm{nm}(\log \varepsilon): 285$ (4.02), 320 (3.55) (sh). CD $\lambda_{\max }$ $(\mathrm{EtOH}) \mathrm{nm}(\Delta \varepsilon): 279(+1.27), 307(-2.58) .{ }^{1} \mathrm{H}-\mathrm{NMR}\left(\mathrm{CD}_{3} \mathrm{OD}\right): \delta: 0.80$ $\left(1 \mathrm{H}, \mathrm{t}, J=7.2 \mathrm{~Hz}, \mathrm{H}-4^{\prime}\right), 1.03\left(1 \mathrm{H}, \mathrm{d}, J=6.9 \mathrm{~Hz}, \mathrm{H}-5^{\prime}\right), 1.28\left(1 \mathrm{H}, \mathrm{m}, \mathrm{H}-3^{\prime} \mathrm{a}\right)$, $1.72\left(1 \mathrm{H}, \mathrm{m}, \mathrm{H}-3^{\prime} \mathrm{b}\right), 3.23-3.44\left(3 \mathrm{H}, \mathrm{m}, \mathrm{H}-2^{\prime \prime}, 3^{\prime \prime}, 4^{\prime \prime}\right), 3.49$ (2H, s, H-5"'), $3.51\left(1 \mathrm{H}, \mathrm{m}, \mathrm{H}-5^{\prime \prime}\right), 3.54$ (1H, dd, $\left.J=3.3,9.6 \mathrm{~Hz}, \mathrm{H}-6^{\prime \prime} \mathrm{a}\right), 3.66$ (1H, d, $J=9.9$ Hz, H-4"'a), 3.79 (1H, m, H-2'), 3.82 (1H, d, $\left.J=2.4 \mathrm{~Hz}, \mathrm{H}-2^{\prime \prime \prime}\right), 3.89$ (1H, d, $\left.J=9.9 \mathrm{~Hz}, \mathrm{H}-4^{\prime \prime \prime} \mathrm{b}\right), 3.94\left(1 \mathrm{H}, \mathrm{dd}, J=3.9,9.6 \mathrm{~Hz}, \mathrm{H}-6^{\prime \prime} \mathrm{b}\right), 4.88(1 \mathrm{H}, \mathrm{d}, J=2.4$ $\left.\mathrm{Hz}, \mathrm{H}-1^{\prime \prime \prime}\right), 5.91$ (1H, d, $\left.J=7.5 \mathrm{~Hz}, \mathrm{H}-1^{\prime \prime}\right), 5.87$ (1H, d, $\left.J=2.4 \mathrm{~Hz}, \mathrm{H}-4\right), 6.08$ $(1 \mathrm{H}, \mathrm{d}, J=2.4 \mathrm{~Hz}, \mathrm{H}-6) .{ }^{13} \mathrm{C}-\mathrm{NMR}$ : see Table 1 . FAB-MS $m / z: 1007(2 \mathrm{M}-$ $\mathrm{H})^{-}, 503(\mathrm{M}-\mathrm{H})^{-}, 209$ (M-glucose-apiose) ${ }^{-}$. Anal. Calcd for $\mathrm{C}_{22} \mathrm{H}_{32} \mathrm{O}_{13}: \mathrm{C}$, 52.38; H, 6.39. Found: C, 52.21; H, 6.37.

Acid Hydrolysis of 3 A solution of $3(5 \mathrm{mg})$ in $1 \mathrm{M} \mathrm{H}_{2} \mathrm{SO}_{4}(2 \mathrm{ml})$ was heated at $80{ }^{\circ} \mathrm{C}$ for $3 \mathrm{~h}$. The reaction mixture was extracted with $\mathrm{Et}_{2} \mathrm{O}$ three times and the combined organic phase was evaporated to dryness under reduced pressure to furnish 2-(2-methylbutyryl)phloroglucinol ${ }^{12)}(2.4 \mathrm{mg}) .{ }^{1} \mathrm{H}-$ NMR (CD $\mathrm{OD}): \delta: 0.90\left(1 \mathrm{H}, \mathrm{t}, J=7.2 \mathrm{~Hz}, \mathrm{H}-4^{\prime}\right), 1.11(1 \mathrm{H}, \mathrm{d}, J=6.6 \mathrm{~Hz}, \mathrm{H}-$ $\left.5^{\prime}\right), 1.38\left(1 \mathrm{H}, \mathrm{m}, \mathrm{H}-3^{\prime} \mathrm{a}\right), 1.79$ (1H, m, H-3'b), 3.85 (1H, m, H-2'), $5.80(2 \mathrm{H}$, $\mathrm{s}, \mathrm{H}-4,6)$. The water layer was neutralized with Amberlite IRA-400 $\left(\mathrm{OH}^{-}\right.$ form) resin, concentrated to dryness and subjected to silica gel chromatography $\left[\mathrm{CHCl}_{3}-\mathrm{MeOH}-\mathrm{H}_{2} \mathrm{O}(6: 4: 1)\right]$ to afford D-glucose $(1.5 \mathrm{mg}):[\alpha]_{\mathrm{D}}^{25}$ $+24.3^{\circ}\left(c=0.12, \mathrm{H}_{2} \mathrm{O}\right)$ and D-apiose $(1.2 \mathrm{mg}):[\alpha]_{\mathrm{D}}^{25}+6.5^{\circ}\left(c=0.09, \mathrm{H}_{2} \mathrm{O}\right)$.

Acknowledgements The authors are grateful to Mr. K. Inada and Mr. N. Yamaguchi (Nagasaki University) for NMR and MS measurements. One of the authors (Y. J. Zhang) is grateful to the Japanese Government for the scholarship awarded her.

\section{References and Notes}

1) Zhang Y.-J., Tanaka T., Yang C.-R., Kouno I., Chem. Pharm. Bull., 49, 537-540 (2001).

2) Zhang Y.-J., Abe T., Tanaka T., Yang C.-R., Kouno I., J. Nat. Prod., 64, $1527-1532$ (2001).

3) Zhang Y.-J., Tanaka T., Iwamoto Y., Yang C.-R., Kouno I., Tetrahedron Lett., 41, 1781-1784 (2000).

4) Zhang Y.-J., Tanaka T., Iwamoto Y., Yang C.-R., Kouno I., J. Nat. Prod., 63, 1507-1510 (2000)

5) Zhang Y.-J., Tanaka T., Iwamoto Y., Yang C.-R., Kouno I., J. Nat. Prod., 64, 870-873 (2001).

6) Wagner H, Chari V. M., Sonnenbichler J., Tetrahedron lett., 21, 17991802 (1976)

7) Markham K. R., Ternal B., Stanley R., Geiger H., Mabry T. J., Tetrahedron, 34, 1389-1397 (1978).

8) Grande M., Piera F., Cuena A., Torres P., Bellido I. S., Planta Med., 51, $414-419$ (1985).

9) Manez S., Paya M., Terencio C., Villar A., Planta Med., 54, 187-188 (1988).

10) Rahman W., Ishratullah K., Wagner H., Seligmann O., Chari V. M., Osterdahl B.-G., Phytochemistry, 17, 1064-1065 (1978).

11) El Sissi H. I., Saleh N. A. M., El Negoumy S. I., Wagner H., Iyengar M. A., Seligmann O., Phytochemistry, 13, 2843-2844 (1974).

12) Kosasi S., Sluis W. G. V. D., Labadie R. P., Phytochemistry, 28, $2439-$ 2441 (1989).

13) Coxon D. T., Holmes A., Ollis W. D., Vora V. C., Tetrahedron, 28, 2819-2826 (1972).

14) Nishizawa M., Yamagishi Y., Nonaka G., Nishioka I., J. Chem. Soc., Perkin Trans. 1, 1983, 961—965 (1983).

15) Haddock E. A., Gupta P. K., Al-Shafi S. M. K., Haslam E., J. Chem. Soc., Perkin Trans. 1, 1982, 2515-2524 (1982).

16) Nishizawa M., Yamagishi Y., Nonaka G., Ageta M., J. Chem. Soc., Perkin Trans. 1, 1982, 2963-2968 (1982).

17) Lin T.-C., Chemical Studies on Tannins and Related Compounds from Combretaceae. Ph.D. Thesis, Kyushu University, Fukuoka, Japan, 1990, pp. $20-21$.

18) Moretti C., Sauvain M., Lavaud, C., Massiot G., Bravo J.-A., Munoz V., J. Nat. Prod., 61, 1390-1393 (1998).

19) Wenkert E., Gottlieb H. E., Phytochemistry, 16, 1811-1816 (1977).

20) Yazaki Y., Hillis W. E., Phytochemistry, 15, 1180-1182 (1976).

21) Ueda M., Okazaki M., Ueda K., Yamamura S., Tetrahedron, 56, $8101-8105$ (2000).

22) Yoshihara T., Omer E.-S. A., Koshino H., Sakamura S., Kikuta Y., Koda Y., Agric. Biol. Chem., 53, 2835-2837 (1989).

23) Gaffield W., Tetrahedron, 26, 4093-4108 (1970).

24) Bohm B. A., "The Flavonoids," ed. by Harborne J. B., Mabry T. J., Mabry H., Academic, New York, 1975, part I, pp. 594-595.

25) Jiang Z.-H., Tanaka T., Kouno I., Chem. Pharm. Bull., 47, 421-422 (1999). 